\title{
Comparative study of the muralytic activity of rumen microbes measured in situ in llamas and sheep
}

\author{
C Kayouli 1, C Dardillat 2, JP Jouany ${ }^{2}$ \\ 1 INAT, laboratoire de Nutrition, 43, av Charles-Nicolle, 1002 Tunis Belvédère, Tunisia; \\ 2 INRA, station de recherches sur la Nutrition des herbivores, centre de recherches de Clermont- \\ Ferrand-Theix, 63122 Saint-Genès-Champanelle, France
}

Recent studies have shown that microbial activity against plant cell walls is greater in the first forestomach of the dromedary than in that of the ruminant (Kayouli et al, 1991).

The aim of the present work was to compare the in situ degradation of a wheat straw in 3 llamas and 3 sheep fed on a mixed diet (grass hay / barley $=88 / 12$ ) at $90 \%$ ad libitum level. The wheat straw was ground and selected by retention on a 100-micron sieve. Nine nylon bags containing the wheat straw were incubated for $72 \mathrm{~h}$ in the rumen of each sheep or each llama, after which 3 bags were distributed between the 3 sheep, 3 others being distributed between the 3 llamas for a further $48 \mathrm{~h}$. The 3 remaining bags were removed,

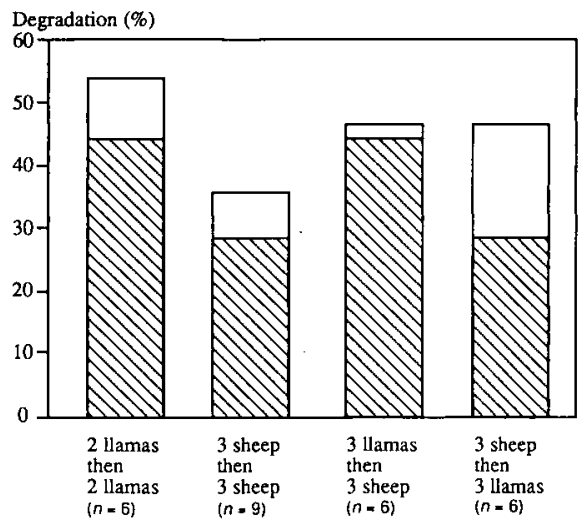

Fig 1. In sacco degradation of the DM of wheat straw in the rumen of llamas and sheep (first incubation of $72 \mathrm{~h}$, followed by the second of $48 \mathrm{~h}$ ). washed, dried and weighed. The extent of $d r y$ matter (DM) loss was measured. One llama was not used during one trial because its rumen cannula had become accidentally detached.

The degradation of the DM of wheat straw after $72 \mathrm{~h}$ (fig 1) was significantly lower in the sheep than in the llamas (29.0 as against $45.2 \%$, respectively). Likewise, after $120 \mathrm{~h}$ of digestion, degradation was higher in the llamas (53.3 as against $36.5 \%$ ). There was no significant improvement in the digestion of the wheat straw that had been incubated for $72 \mathrm{~h}$ in the llamas when it was placed for a further $48 \mathrm{~h}$ in the sheep rumen ( 47.7 as against $45.2 \%)$. In contrast, the degradation of the DM of wheat straw from the sheep rumen was greatly increased when the nylon bags were then placed in the first compartment of the llama (47.7 vs $29.0 \%$ ). The extent of degradation was exactly the same $(47.7 \%)$ in samples inoculated successively in both animals, and in no matter which order (sheep + llama or llama + sheep).

In conclusion, the muralytic activity of the microbial population of the forestomachs of llamas against a straw with low digestibility is therefore much higher than that of the sheep rumen.

Kayouli C, Jouany JP, Ben Amor J (1991) Anim Feed Sci Technol 33, 237-245 